\title{
Efficient and Accurate Target Sensor Tracking in Wireless Sensor Networks
}

\author{
Suman Jyothula ${ }^{1}$, Patan Shakira ${ }^{2}$ \\ I'Assistant Professor, CSE Dept., India) \\ ${ }^{2}$ (II M.Tech, CSE Dept., India)
}

\begin{abstract}
In the tracking scheme illustrated, where the sensors are deployed in a triangular fashion in a hexagonal mesh such that the hexagon is divided into a number of equilateral triangles. Where the technique is used for detection is the trilateration technique in which intersection of three circles is used to determine through the object location. While the object was tracked by the three sensors, distance where it from a fourth sensor and it is also being calculated simultaneously. Here the difference is that closest of three sensors detect at a frequency of one second while the fourth sensor detects the object location at twice the frequency. By using the distance information from the fourth sensor and a simple mathematical technique, location of object is to be predicted for every half second as well. Where the key thing that is to note the forth sensor node is not used for detection but only for estimation of the object at half second intervals and hence does not utilizes much power. By using these techniques, tracking capability of the system is increased.
\end{abstract}

Keywords: Application-Specific, Data-Centric, Negotiation Based Protocols, Target Sensor Tracking.

\section{Introduction}

MOBILITY management has long been recognized as a major challenge in mobile ad hoc networks (MANETs). A MANET generally has the following characteristics:

1. Firstly a New members can also join and can leave the network any time,

2. Here no base station was available for providing connectivity to backbone hosts or to other mobile hosts,

3. It is very difficult in implementing the sophisticated scheme for the handover and location management,

4. Each node acts as a router, forwarding packets from others nodes, and

5. Communication connectivity is usually "weak" in the sense that it is easily broken due to node movement

Wireless communication and MEMS - the two technologies which have revolutionized the way we live have also resulted in the development of wireless sensor networks. They comprise of relatively inexpensive sensor nodes capable of collecting, processing them, storing them and transferring the information from one node to another node. These nodes can be able to manage autonomously form a network through which sensor readings can also be propagated. Since here the sensor nodes have some intelligence, where the data can be processed as it flows through the network. Where the latter is to be done wirelessly these days using some networking principles. The flexibility of such installation and configuration has greatly improved resulting in a flurry of research activities commencing in the field of sensor networks owing to their ready acceptance in various industries which contains the security, telecommunications and also automobile to name a few.

Recently, there has been growing interest in the deployment of multiple mobile sensors for target tracking applications. For example, progress in the development of unmanned terrestrial or aerial vehicles and some of their specific advantages have rapidly enhanced their use in applications such as search, surveillance and adversarial target tracking.

The sensors can be deployed in any facility or area which has to be sensed in 3 main types. It will be either 1) triangular sensor deployment, 2) square sensor deployment or 3) irregular sensor deployment. Such deployments are to be depicted as follows:

During a relief operation or at a disaster site, when the need is to know what the conditions are and we need to perform i.e. to scan the area, where the sensors can be dropped from a helicopter over the area. In which they help in scanning the area and construct the maps which are very helpful for first responders and assist them in performing their job. Right now, however the process is very human intensive

We consider that this sensor network with that of two mobile sensors with which having a two-way communication link with the fusion center. The goal of the fusion center is to track a moving target by controlling the mobile sensors that interactively provide the fusion center with a partial view of the target. Specifically, when the target is in the sensing range of either sensor, the position of the target is relayed to the fusion center. Using this information, the fusion center interactively steers the two sensors to achieve optimal 
tracking of the target in the sense of minimizing a linear combination of the tracking cost and the energy cost for steering the sensors the (uncontrolled) moving target and the (controlled) mobile sensors; they are coupled through the observation made available to the controlling agent (the fusion center).

To capture all aspects of such tracking problems in real applications, one needs full descriptions of both the uncontrolled dynamics of the target as well as the controlled dynamics of the mobile sensors. In the applications of interest, sensors are often placed on mobile vehicles; hence, the control system describing a mobile sensor is in itself a complicated non-linear system with a large state space.

As a consequence, a complete analysis of the whole tracking problem that comprises this complicated system interacting in a feedback loop with the target's dynamics is apparently a daunting task

In this paper, we present an efficient mobility management scheme that can be implemented in a fully distributed manner. With which the proposed mobility management scheme is a general framework that incorporates both the positive and negative consequences of node movement; it allows a node to autonomously decide whether it should move and where it should move to. It is based on concepts taken from Bayesian estimation theory.

\section{Related Work}

Since, sensor network requirements are different enough from those of traditional wired and wireless networks, where it is important in considering a design having the following features:

1.1 a) Data-Centric: Unlike traditional networks, a sensor node may not need an identity (e.g., an address). Rather, applications focus on the data generated by the sensors. In some situations, consider an example, for the querying a specific faulty sensor, the ability to address an individual sensor is clearly necessary. Data is named by attributes and applications request data matching certain attribute values. Most sensor data is associated with the physical context of the phenomena being sensed. Hence, spatial coordinates are a natural way to name data. This makes localization - determination of the position of the node in some coordinate system - an important problem.

b) Application-Specific: Sensor networks can be designed to perform a number of functions. Individual sensor nodes in a network can perform the functions of information/data gathering, collecting data and storing and forwarding of information/data on request from known neighboring nodes. Where this is in contrast for a centralized structure in the case of routers that facilitate node-to-node packet switching in traditional networks. Individual sensors report their data to a central node, Then that will performs the computation required for the application. Where this centralized structure is definitely bad choice for several reasons: it provides a single point of failure, so that it can be energy inefficient, and also it doesn't scale for large networks.

Thus, localized algorithms are better suited to sensor networks in which each sensor node communicates with neighboring nodes and computation is operated locally, yet the total/entire structure achieves a desired global objective. Finally the sensors are physically distributed, so that it is not unnatural to design sensor networks using distributed algorithms.

\subsection{Routing}

Conventional routing protocols are not very energy efficient. In the case of sensor networks, the nodes have very limited energy and there is no external source of battery input power. Hence, care must be taken as to how the information routing takes place. It should be such that there is a fine balance between shortest path selection and energy used. Routing protocols are either proactive or reactive.

In the former, updated routing information between all nodes is maintained in the routing tables. In the latter, the routes are created as and whenever they are to be needed. In any case, routing can considered to be either forward based (source initiated) or backward based (destination initiated). The simplest case of routing is flooding in which a node sends the information to all its neighboring nodes. However and whenever this scheme will suffers from the following problems:

a) Implosion: If a node is a neighbor to nodes holding the same information, it will have duplicate copies of the same data resulting in a waste of resources.

b) Resource Management: In this scheme, the nodes are not resource-aware and perform their activities regardless of the available energy. This is highly undesirable in a sensor network scenario.

The problem of tracking targets with sensor networks has received attention from various angles. In [6], the authors consider the case where a set of $\mathrm{k}$ targets need to be tracked with 3 sensors per target from the resource requirement viewpoint. This shows that the probability that all targets can be assigned 3 unique sensors shows phase transition properties as the level of communication between the sensors increases. In an information driven sensor collaboration mechanism is proposed.

In this mechanism, measures of information utility are utilized to decide future sensing actions. Collaborative signal processing aspects for target classification in sensor networks is addressed in [7]. Tracking based on relations in the targets is discussed in [13]. 
Techniques for locating targets using a variety of mechanisms have been proposed in [5], [11], [10]. However, current literature does not address the issue of a scalable architecture for coordinating a sensor network for the purpose of target tracking. Nor is there any existing work which deals with the feasibility, minimization of computation and communication overheads and understanding the tradeoffs in such systems. In this paper we address these issues.

\subsubsection{Negotiation Based Protocols}

These protocols are called SPIN (Sensor Protocols for Information via Negotiation) protocols. As the name suggests, there is negotiation prior to transfer of date between them. Meta-data are information descriptors here and used to eliminate the transmission of redundant in data. In SPIN, track was kept of the energy level of the node. Prior to transmission, a node checks with it as to whether it has sufficient energy to carry out the transmission of that data. The three main types of messages passed between nodes in this family of protocols are:

ADV: Whenever a node has some new data, it sends this message advertising to its neighbors. The message containing the meta-data as well.

REQ: Whenever a node wishes to receive some of the data, it sends out of this message.

DATA: This message contains the actual data along with a meta-data header. The following protocols constitute the SPIN family of protocols:

a) SPIN-PP: This is a point-to-point communication protocol. In this, energy is not a constraint. The data is transmitted by the source as many times as there are requesting nodes and hence is very energy inefficient. It is thus not ideal for a wireless sensor network scenario. Whenever the node had some data, in which it sends out an ADV message.

The neighboring node checks the meta-data header to see whether it has the data already that was stored. If not, then it was sends out a REQ message requesting for then that of data item. Upon receiving these messages, the originating node sends DATA message containing the data item. Thus, this will be surely a 3-way handshake protocol. The advantage in this protocol is that a node needs to know only about its single hop neighbors.

b) SPIN-EC: This is also a 3-way handshake protocol but has energy constraints. A node participates in the protocol only if its energy level is greater than the energy threshold, i.e. it can complete all stages of the protocol.

c) $\boldsymbol{S P I N - B C}$ : This protocol is for broadcast networks. The nodes use a single shared channel for communication purposes. Whenever a node which sends a message, all the nodes within a range receive that message. If the originating node receives a request it broadcasts the data item only once, even though it may have got multiple requests. In this if other node receives a REQ message, it cancels that request so there are no redundant requests for the same data item. Also, whenever a node receives an ADV message it cannot immediately respond with an REQ message and has to wait for a certain time before doing so.

d) SPIN-RL: This is primarily an extension of the above protocol. In this, each node keeps track of all the advertisements it hears and the nodes it hears them from. If the node does not receive the requested data within a certain period of time, it sends out the request again. Where the originating nodes having a restriction on the frequency at which they can send the data messages

\section{Proposed Method}

We are focusing on We are currently working on more complicated prediction algorithms in order to achieve lower miss rates for a given tracking resolution. Additional work can also be conducted on interpolation mechanisms at cluster heads when a sensor reports multiple targets simultaneously. The relationship between energy consumed and prediction accuracy also remains an open issue. Another possible direction of work is to accommodate mobile sensors. With mobile sensors the scenario complicates considerably and significantly different approaches to the solution may be necessary networks.

In this section we describe our proposed protocol for efficiently tracking mobile targets with sensor

The Distributed Prediction Tracking (DPT) algorithm is specifically aimed at addressing the various challenges outlined in the Section II while accurately tracking moving targets. As the name suggests, this algorithm does not require any central control point, eliminating the possibility of a single point of failure and making it robust against random node failures. The tracking task is carried out distributively by sequentially involving the sensors located along the track of the moving target.

Our algorithm assumes a cluster based architecture for the sensor network and the choice was motivated by the need to ensure the sensor network's scalability and energy efficiency. A different number of clustering algorithms have been proposed in literature ([1], [8] and the references therein) and in this paper, we are not at all concerned with that of the details of the clustering mechanisms. Any suitable clustering mechanism 
from those proposed in literature may be used and we note that our protocol does not impose any specific require

\section{Assumptions Of The DPT Algorithm}

We now describe the assumptions made by the DPT algorithm. While no assumption is made on the choice of the clustering algorithm, we assume that the Cluster Head $(\mathrm{CH})$ has the following information about all sensors belonging to its cluster: (1) Sensor identity, (2) Location and (3) Energy level. When tracking a moving target and deciding which sensors to use for tracking, the cluster head's decision-making procedure will be based on this information.

The system consists of a large number of sensor nodes distributed over the region of interest. The distribution of the nodes is uniform to obtain maximum coverage of the entire region nodes. Then the entire region of interest is divided into a number of clusters. Each cluster has a Cluster Head $(\mathrm{CH})$ and several normal nodes $(\mathrm{NN})$.

The cluster heads perform computationally intensive tasks in that the cluster. Here the normal nodes are capable of sensing seismic, acoustic and also the magnetic activity. Where it is assumed that there is a high probability of targets originating outside the region of interest and entering through that periphery, therefore, here nodes with the higher capabilities are deployed at the periphery. Cluster heads then find out the neighboring nodes by broadcasting information to all the nodes in the close proximity of the cluster heads. The target originates from outside the region of interest and enters then to the coverage area, through that the periphery. It was assumed that there is a high probability of targets originating outside the region of interest and entering through the periphery, therefore, that the nodes with higher capabilities are deployed at the periphery.

Sensors transmit the sensed information using a single-hop or a multi-hop path to the base station. The base station processes the sensed data to determine the targets path, once the target is to be detected in a particular cluster or, a track manager is assigned and follows the target in that of the cluster.

The base station processes the sensor data, determines the target's trajectory and may provide the navigation guidance to the friendly object. The selection of sensors is dependent on the target type and the signature emitted by the target. For this paper, we assume that the sensors have both the detection as well as ranging capacity and the same is available to sensor node. A combination of passive infrared sensors and ultrasonic ranging sensors can be used to for object detection.

This forms the essential idea behind the DPT algorithm. We now introduce and explain the main components of the algorithm.

Target Descriptor Formulation Algorithm: In order to identify the target and provide the target's location information, cluster heads use a Target Descriptor (TD). The following items are incorporated in the TD:

1) Target identity

2) Target's present location

3) Target's next predicted location

4) Time stamp

\section{Conclusion}

In this paper we proposed a feasible solution for distributed tracking of mobile targets using sensor networks: the Distributed Predictive Tracking algorithm. DPT's essential idea is to predict the target's future location based on known previous locations. Since it is totally distributed, it scales well without having any central point of failure and can be easily extended to tracking in 3-dimension.

\section{References}

[1] S. Banerjee and S. Khuller, "A clustering scheme for hierarchical control in multi-hop wireless networks," Proceedings of IEEE INFOCOM, pp. 1028-1037, Anchorage AK, April 2001.

[2] D. Estrin, L. Girod, G. Pottie and M. Srivastava, "Instrumenting the world with wireless sensor networks," Proceedings of ICASSP, pp. 2033-2036, Salt Lake City, UT, May 2001.

[3] D. Estrin, R. Govindan, J. Heidemann and S. Kumar, "Next century challenges: Scalable coordination in sensor networks," Proceedings of ACM/IEEE MOBICOM, pp. 263-270, Seattle, WA, August 1999.

[4] J. M. Kahn, R. H. Katz and K. S. J. Pister, "Mobile networking for smart dust," Proceedings of ACM/IEEE MOBICOM, pp. 271 278, Seattle, WA, August, 1999.

[5] L. M. Kaplan, Q. Le and P. Molnar, "Maximum likelihood methods for bearings-only target localization," Proceedings of IEEE ICASSP, pp. 554-557, Salt Lake City, UT, May, 2001.

[6] B. Krishnamachari, S. B. Wicker and R. Bejar, "Phase transition phenomena in wireless ad-hoc networks," Proceedings of IEEE GLOBECOM, San Antonio, TX, November 2001.

[7] D. Li, K. Wong, Y. H. Hu and A. Sayeed, "Detection, classification and tracking of targets in distributed sensor networks," IEEE Signal Processing Magazine, vol. 19, no. 2, March 2002. 
[8] C. R. Lin and M. Gerla, “Adaptive clustering for mobile wireless networks," IEEE Journal of Selected Areas in Communications, vol. 15 , no. 7 , pp. 1265-1275, July, 1997.

[9] K. Sohrabi, J. Gao, V. Ailawadhi and G. J. Pottie, "Protocol for selforganization of a wireless sensor network," IEEE Personal Communications Magazine, vol.7, no.5, pp. 16-27, October 2000.

[10] J. S. Scholl, L. P. Clare and J. R. Agre, "Wavelet packet-based target classification schemes," Meeting of IRIS Specialty Group on Acoustic and Seismic Sensing, Laurel, MD, September, 1998.

[11] K. Yao, R. E. Hudson, C. W. Reed, T. L. Tung, D. Chen and J. C. Chen, "Estimation and tracking of an acoustic/seismic source using a beamforming array based on residual minimizing methods," Proceedings of IRIA-IRIS, pp. 153-163, January 2001.

[12] F. Zhao, J. Shin and J. Reich, "Information-driven dynamic sensor collaboration for target tracking," IEEE Signal Processing Magazine, vol.19, no. 2, pp. 61-72, March 2002

[13] L. Guibas, "Sensing, tracking and reasoning with relations," IEEE Signal Processing Magazine, vol. 19, no. 2, pp. 73-85, March 2002.

\section{ABOUT AUTHOR}

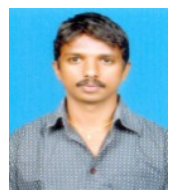

SUMAN JYOTHULA received the B.Tech degree in 2009 from JNTU, Kakinada, and the M.E degree in 2011 from Sathyabama University; He is currently working toward the Ph.D. degree in Acharya Nagarjuna University. His research interests include Networking, Cloud Computing, and cryptography.

PATAN SHAKIRA received the B.Tech degree in Information Technology from JNTU Kakinada, in 2012.She is currently working toward the Master Degree at the JNTU Kakinada. Her research interests include Image processing, Data hiding, and also networking. 